\title{
Comunicação
}

\section{Características físico-químicas de cultivares de macieiras pouco exigentes em frio ${ }^{1}$}

\author{
Dili Luiza de Oliveira ${ }^{2}$, Angelo Albérico Alvarenga ${ }^{3}$,Emerson Dias Gonçalves ${ }^{4}$, Marcelo Ribeiro Malta $^{5}$
}

\section{RESUMO}

O sul do Brasil é a região brasileira com mais tradição na produção de maçãs. Contudo, novos cultivares menos exigentes em frio estão viabilizando os cultivos em outras regiões do Brasil, como no Estado de Minas Gerais, onde a cultura encontra-se em franca expansão. Os atributos físico-químicos dos frutos são importantes, tanto para a industrialização, quanto para o consumo dos frutos in natura. Neste sentido, objetivou-se, com este trabalho, realizar a caracterização físico-química de maçãs de oito cultivares, pertencentes à coleção estabelecida na Serra da Mantiqueira, sul de Minas Gerais. O experimento foi conduzido em pomar experimental, na Empresa de Pesquisa Agropecuária de Minas Gerais - EPAMIG, na cidade de Maria da Fé, sul de Minas Gerais. Foram analisados frutos de macieira de oito cultivares (Michal, Ein Shemer, Soberana, Galícia, Eva, IAC 2079, Condessa e Anna) oriundos de plantas com seis anos de idade. Foram avaliados: massa do fruto, diâmetro transversal, diâmetro longitudinal, firmeza de frutos, acidez titulável (AT), sólidos solúveis (SS), relação SS/AT, pH e produção de frutos. Os cultivares 'Anna' e 'Condessa' produziram os maiores frutos; 'Eva' apresentou maior produção e frutos mais firmes; 'Anna', os mais macios; enquanto 'Galícia' e 'IAC 2079,' os de sabor mais equilibrado.

Palavras-chave: Malus sp., cultivares, qualidade de frutos.

\section{ABSTRACT}

\section{Apple cultivars undemanding as to cold and fruit quality}

Southern Brazil is a traditional apple-growing region. However, new less cold-demanding cultivars are making the cultivation of apples viable in other Brazilian regions, such as in the state of Minas Gerais, where the crop is in a remarkable expansion. The quality attributes of the fruits are important both to the industrialization and the fresh consumption. In this sense, this work was aimed at characterizing fruits of different less-cold demanding apple cultivars established on the Mantiqueira Mountains, South of Minas Gerais. The experiment was conducted in an experimental orchard in Empresa de Pesquisa Agropecuária de Minas Gerais - EPAMIG (Minas Gerais Agricultural Research Company - EPAMIG) in the municipality of Maria da Fé - south of Minas Gerais. Fruits of of eight apple cultivars from six-yearold plants were evaluated (Michal, Ein Shemer, Soberana, Galícia, Eva, IAC 2079, Condessa and Anna). Fruit mass, transversal diameter, longitudinal diameter, fruit firmness, production, total acidity (TA), total soluble solids (TSS), TA/ TSS ratio, $\mathrm{pH}$ and fruit yield were recorded for each cultivar. The cultivars Anna and Condessa yielded the largest fruits; cv. Eva produced the firmest and the highest yielding cultivar; cv. Anna produced the softest fruits, while Galicia and IAC 2079 produced fruits of the most balanced flavor.

Key words: Malus sp, cultivars, fruit quality.

\footnotetext{
Recebido para publicação em 18/10/2012 e aprovado em 26/06/2013.

${ }^{1}$ Fontes financiadoras: FAPEMIG / CNPq.

${ }^{2}$ Bióloga, Doutora. Empresa de Pesquisa Agropecuária de Minas Gerais, s/n, Campus da UfLA, Avenida Inácio Valentim, s/n, 37200-000, Lavras, Minas Gerais, Brasil. dililuiza@gmail.br (autora para correspondência).

${ }^{3}$ Engenheiro-Agrônomo, Doutor. Empresa de Pesquisa Agropecuária de Minas Gerais, s/n, Campus da UfLA, Avenida Inácio Valentim, s/n, 37200-000, Lavras, Minas Gerais, Brasil. angelo@epamig.br

${ }^{4}$ Engenheiro-Agrônomo, Doutor. Empresa de Pesquisa Agropecuária de Minas Gerais, Fazenda Experimental de Maria da Fé, Rua Wahington Alvarenga Viglioni, s/n, Bairro Vargedo, Zona Rural, 37517-000, Maria da Fé, Minas Gerais, Brasil. emerson@epamig.br

${ }^{5}$ Engenheiro-Agrônomo, Doutor. Empresa de Pesquisa Agropecuária de Minas Gerais, s/n, Campus da UFLA, Avenida Inácio Valentim, s/n, 37200-000, Lavras, Minas Gerais, Brasil.marcelomalta@epamig.br
} 


\section{INTRODUÇÃO}

O sul do Brasil é a região brasileira com mais tradição na produção de maçãs, nos Estados do Paraná, Santa Catarina e Rio Grande do Sul. Contudo, novos cultivares menos exigentes em frio estão viabilizando os cultivos em outras regiões do Brasil, como no Estado de Minas Gerais, onde a cultura encontra-se em franca expansão (Oliveira et al., 2011). A pomicultura em Minas Gerais, mais especificamente na Serra da Mantiqueira, sul do Estado, é recente e existem poucos dados de pesquisa sobre a qualidade dos frutos dos cultivares recomendados para o cultivo em regiões de inverno mais ameno.

As características físicas, como tamanho e cor do fruto, influenciam diretamente a aceitação pelo consumidor e o rendimento industrial, ao passo que as características intrínsecas, como os teores de sólidos solúveis, os quais representam indiretamente os açúcares e acidez titulável, bem como o balanço entre essas variáveis, são indicadores das características organolépticas, importantes, tanto na industrialização, quanto no consumo dos frutos in natura (Alvarenga \& Fortes, 1985).

Por essas razões, objetivou-se, neste trabalho, realizar a caracterização físico-química de maçãs de oito cultivares, pertencentes à coleção estabelecida na Serra da Mantiqueira, sul de Minas Gerais.

\section{MATERIAL E MÉTODOS}

O experimento foi conduzido em pomar experimental, na Empresa de Pesquisa Agropecuária de Minas Gerais EPAMIG, na cidade de Maria da Fé, que representa bem a região, caracterizada por inverno ameno, em comparação com o da tradicional região produtora de maçãs do sul do Brasil.

Maria da Fé está localizada na microrregião da Serra da Mantiqueira, no sul do Estado de Minas Gerais, a $22^{\circ}$ 18 ' S e 45 23' O, em altitude média de $1276 \mathrm{~m}$. O clima da região é classificado como subtropical de altitude, com inverno seco e verão ameno - Cwb, apresentando temperatura média anual de $17^{\circ} \mathrm{C}$.

Foram analisados frutos de macieira de oito cultivares ('Michal', 'Ein Shemer', 'Soberana', 'Galícia', 'Eva', 'IAC 2079', 'Condessa' e 'Anna'), oriundos de plantas com seis anos de idade, que se encontram em espaçamento de cinco metros entre linhas e dois metros entre plantas. Cada cultivar correspondeu a um tratamento. O delineamento experimental utilizado foi inteiramente casualizado, com cinco repetições e uma planta por parcela, em esquema unifatorial, sendo o fator de tratamento os cultivares. Foram coletados cinco frutos de cada árvore, obtendo-se uma amostra composta para a determinação dos dados físico-químicos.

Foram avaliados os dados físicos: massa de frutos (g), por pesagem em balança de precisão dos frutos colhidos
; diâmetro transversal ( $\mathrm{mm}$ ) e diâmetro longitudinal ( $\mathrm{mm}$ ), medidos com paquímetro digital; firmeza de frutos, com penetrômetro digital, tomando-se duas leituras por fruto, em lados opostos de sua região equatorial, e produção de plantas ( g planta $^{-1}$ ) e os dados químicos: acidez titulável, realizada por titulometria; sólidos solúveis, com refratômetro digital, expressando-se os resultados em ${ }^{\circ}$ Brix, e $\mathrm{pH}$, por leitura em pHmetro.

Os dados foram submetidos à análise de variância e as médias agrupadas pelo teste de Scott-Knott, a 5\% de probabilidade.

\section{RESULTADOS E DISCUSSÃO}

Com relação à massa de frutos, observa-se que os cultivares 'Condessa' e 'Anna' obtiveram as maiores médias (128,15 e 141,72 g), seguidos de 'IAC 2079', 'Eva' e 'Galícia'. Os que apresentaram menores massas foram 'Ein Shemer', 'Michal' e 'Soberana' (Tabela 1). Segundo dados do Ceasa (Almeida \& Alves, 2012), cada fruto é classificado segundo sua massa, o que determina o seu calibre. $\mathrm{O}$ calibre é um dos principais fatores que definem a qualidade de frutos, determinando, assim, seu preço de mercado (Almeida \& Alves, 2012).

Para diâmetro longitudinal dos frutos, os maiores valores foram observados no grupo formado pelos cultivares 'Anna', 'Galícia' e 'Condessa' (Tabela 1). Os menores diâmetros longitudinais foram observados nos frutos dos cultivares 'Soberana', 'Ein Shemer' e 'Michal'.

Quanto ao diâmetro transversal de seus frutos, os cultivares dividiram-se em dois grupos (Tabela 1). O maior valor observado verificou-se no grupo formado pelos cultivares ‘Anna', 'Condessa', 'IAC 2079' e 'Eva', enquanto o menor valor foi observado em grupo formado pelos cultivares 'Michal', 'Ein Shemer', 'Soberana' e 'Galícia'.

Como pode ser observado, o cultivar 'Galícia' apresentou frutos com maior diâmetro longitudinal, porém mais finos (menor diâmetro transversal). Esse formato alongado de frutos de maçã está possivelmente, ligado a um aumento da amplitude térmica encontrado em Maria da Fé. Segundo Westwood (1982), um significativo aumento da amplitude térmica influencia no formato de frutos de maçã.

Para firmeza, valores bem distintos foram observados para os frutos dos oito cultivares (Tabela 1). O cultivar 'Eva' foi o que apresentou o maior valor, sendo seus frutos, na época da colheita, os mais firmes. Os cultivares mais macios foram 'IAC 2079' e 'Ein Shemer' . Resultados semelhantes foram encontrados por Antoniali et al. (2010), por cujos resultados o cultivar 'Eva', foi o que apresentou maior firmeza de frutos, dentre os outros três.

Para acidez titulável, os maiores valores foram observados para frutos dos cultivares 'Anna' e 'Ein Shemer', que são os mais ácidos, enquanto os cultivares 'Galícia' e 
'IAC 2079', apresentaram frutos com os menores valores (Tabela 2).

Os teores de sólidos solúveis variaram entre 10,66 e 13,60 ${ }^{\circ}$ Brix, sendo que os frutos do cultivar 'Michal' apresentaram o valor mais elevado e, os de 'Eva', o valor mais baixo, apesar de não apresentarem diferenças estatísticas significativas (Tabela 2). Porém, o maior valor da relação SS/AT foi verificado em frutos dos cultivares 'Galícia' e 'IAC 2079', indicando melhor sabor (Tabela 2). Segundo Fachinello (1996), a relação SS/AT é um importante indicativo do sabor, pois relaciona os açúcares e os ácidos do fruto. Durante o período de maturação, a relação SS/AT tende a aumentar, por causa da diminuição dos ácidos e do aumento dos açúcares, sendo que o valor absoluto depende do cultivar utilizado.

Quanto ao $\mathrm{pH}$, os maiores valores foram observados em frutos dos cultivares 'IAC 2079', 'Condessa' e 'Galícia', seguidos pelos frutos dos outros cinco cultivares.

Para produção de plantas, os maiores valores foram observados para o cultivar 'Eva', em torno de 3.500 g plan$\mathrm{ta}^{-1}$ (Figura1). Os menores valores foram observados para os cultivares 'IAC 2079', 'Michal', 'Soberana' e a 'Ein

Tabela 1. Massa de frutos (g), diâmetro longitudinal (mm), transversal (mm) e firmeza de frutos (N) de maçãs em Maria da Fé. Empresa de Pesquisa Agropecuária de Minas Gerais - EPAMIG, MG, 2012

\begin{tabular}{lcccc}
\hline Cultivares & Massa & DL & DT & Firmeza \\
\hline Michal & $66,80 \mathrm{c}$ & $50,71 \mathrm{c}$ & $52,42 \mathrm{~b}$ & $33,61 \mathrm{c}$ \\
Ein Shemer & $67,83 \mathrm{c}$ & $53,02 \mathrm{c}$ & $52,77 \mathrm{~b}$ & $25,17 \mathrm{~d}$ \\
Soberana & $58,29 \mathrm{c}$ & $49,94 \mathrm{c}$ & $53,17 \mathrm{~b}$ & $37,60 \mathrm{c}$ \\
Galícia & $103,22 \mathrm{~b}$ & $69,11 \mathrm{a}$ & $56,60 \mathrm{~b}$ & $48,78 \mathrm{~b}$ \\
Eva & $122,47 \mathrm{~b}$ & $59,77 \mathrm{~b}$ & $62,56 \mathrm{a}$ & $56,39 \mathrm{a}$ \\
IAC 2079 & $117,76 \mathrm{~b}$ & $61,22 \mathrm{~b}$ & $62,76 \mathrm{a}$ & $30,14 \mathrm{~d}$ \\
Condessa & $128,15 \mathrm{a}$ & $67,33 \mathrm{a}$ & $63,09 \mathrm{a}$ & $44,02 \mathrm{~b}$ \\
Anna & $141,72 \mathrm{a}$ & $74,39 \mathrm{a}$ & $67,24 \mathrm{a}$ & $37,96 \mathrm{c}$ \\
\hline CV $(\%)$ & 13,32 & 8,49 & 8,54 & 15,90
\end{tabular}

Médias seguidas de mesma letra pertencem ao mesmo grupo pelo teste Scott-Knott a 5\% probabilidade.

Tabela 2. Acidez titulável (g $100 \mathrm{~mL}^{-1}$ de ácido málico), sólidos solúveis ( ${ }^{\circ}$ Brix), pH e relação entre sólidos solúveis e acidez titulável (SS/AT) de frutos de maçã em Maria da Fé. Empresa de Pesquisa Agropecuária de Minas Gerais - EPAMIG, MG, 2012

\begin{tabular}{lcccc}
\hline Cultivares & Acidez titulável & Sólidos solúveis & pH & SS/AT \\
\hline Michal & $4,75 \mathrm{~b}$ & $13,60 \mathrm{a}$ & $3,05 \mathrm{~b}$ & $2,86 \mathrm{c}$ \\
Ein Shemer & $5,46 \mathrm{a}$ & $11,96 \mathrm{~b}$ & $2,95 \mathrm{~b}$ & $2,21 \mathrm{~d}$ \\
Soberana & $4,19 \mathrm{c}$ & $13,42 \mathrm{a}$ & $3,03 \mathrm{~b}$ & $3,21 \mathrm{~b}$ \\
Galícia & $2,92 \mathrm{~d}$ & $12,12 \mathrm{~b}$ & $3,38 \mathrm{a}$ & $4,15 \mathrm{a}$ \\
Eva & $3,82 \mathrm{c}$ & $10,66 \mathrm{c}$ & $3,21 \mathrm{~b}$ & $2,79 \mathrm{c}$ \\
IAC 2079 & $2,92 \mathrm{~d}$ & $12,08 \mathrm{~b}$ & $3,48 \mathrm{a}$ & $3,21 \mathrm{a}$ \\
Condessa & $3,78 \mathrm{c}$ & $11,80 \mathrm{~b}$ & $3,34 \mathrm{a}$ & $3,12 \mathrm{~b}$ \\
Anna & $5,39 \mathrm{a}$ & $12,52 \mathrm{~b}$ & $3,05 \mathrm{~b}$ & $2,32 \mathrm{~d}$ \\
\hline CV $(\%)$ & 8,22 & 4,32 & 4,15 & 9,28 \\
\hline
\end{tabular}

Médias seguidas de mesma letra pertencem ao mesmo grupo pelo teste Scott-Knott a 5\% probabilidade

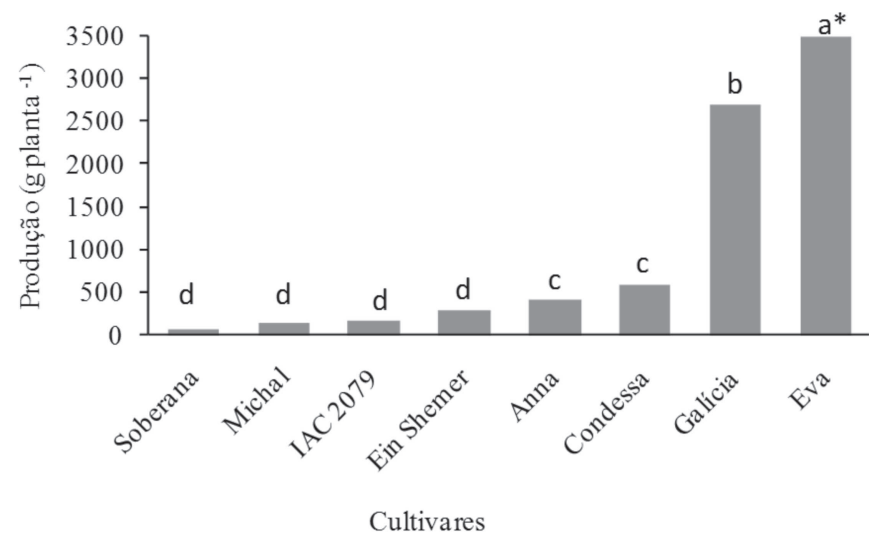

Figura 1. Produção $\left(\mathrm{g}\right.$ planta $\left.^{-1}\right)$ de cultivares de maçã em pomar experimental. Maria da Fé - MG, 2012. Médias seguidas de mesma letra pertencem ao mesmo grupo pelo teste Scott-Knott a 5\% probabilidade. 
Shemer'. Embora as produções tenham sido relativamente baixas, o cultivar 'Eva' destaca-se, frente aos demais cultivares. Com baixo requerimento em frio, esse cultivar é o mais plantado em Minas Gerais, apresentando, em plantios comerciais, produtividade média de $40 \mathrm{t} \mathrm{ha}^{-1}$ (Oliveira et al., 2011), que pode ser atribuída ao manejo adequado empregado pelos produtores.

\section{CONCLUSÕES}

Os oito cultivares de macieiras introduzidos no sul de Minas Gerais, na região da Serra da Mantiqueira Mineira, destacaram-se pelas seguintes características:

Maiores frutos: 'Anna' e 'Condessa';

Frutos mais firmes e maior produção: 'Eva';

Frutos mais macios: 'Anna'; 'Soberana' e 'Michal'

Frutos com sabor mais equilibrado: 'Galícia' e 'IAC 2079'.

\section{REFERÊNCIAS}

Almeida G. V. B. \& Alves A. A. (2012) Mercado de Maçã: Situação atual, ameaças, oportunidades e estratégias para o futuro. Disponível em: <http://www.todafruta.com.br/ portal/icNoticiaAberta.asp?idNoticia $=12448>$. Acessado em: 10 de setembro de 2012.

Alvarenga LR \& Fortes JM (1985) Cultivares de fruteiras de clima temperado. Belo Horizonte, EPAMIG. p.03-11, (Informe Agropecuário, 11).

Antoniali S (2010) Qualidade físico-química de cultivares de macieira na região do leste paulista. In: Congresso Brasileiro de Fruticultura, Jaboticabal. Anais, Sociedade Brasileira de Fruticultura. p.47-48.

ABPM - Associação Brasileira de Produtores de Maçã (2012). Disponível em: <http://www.ceasaminas.com.br/agroqualidade/ maca.asp>. Acessado em: 10 de setembro de 2012.

Fachinello JC, Nachigal JC \& Kersten E (1996) Fruticultura: Fundamentos e práticas. $\quad 1^{\mathrm{a}}$ ed. Pelotas, UFPel. 311p.

Oliveira DL, Alvarenga AA, Gonçalves ED, Abrahão E, Zambon CR \& Norberto PM (2011) Maçã 'Eva' desponta a produção no estado de Minas Gerais. Belo Horizonte, EPAMIG. 4p. (Circular Técnica, 141).

Westwood, N.M. (1982) Fruticultura de zonas temperadas, Madrid. Mundiprensa, $442 \mathrm{p}$. 